\title{
The Net2Plan-OpenStack Project: IT Resource Manager for Metropolitan SDN/NFV Ecosystems
}

\author{
M. Garrich ${ }^{1}$, M. Hernández-Bastida ${ }^{1}$, C. San-Nicolás-Martínez ${ }^{1}$, F. J. Moreno-Muro ${ }^{1}$, P. Pavon-Marino ${ }^{1,2}$ \\ ${ }^{1}$ Universidad Politécnica de Cartagena, Cuartel de Antiguones, Plaza del Hospital 1, 30202 Cartagena, Spain \\ ${ }^{2}$ E-lighthouse Networks Solutions, Calle Ángel s/n, 30202 Cartagena, Spain \\ Email: miquel.garrich@upct.es
}

\begin{abstract}
We demonstrate an open-source Net2Plan extension interfacing multiple OpenStack instances for enabling multi-datacenter IT resource management, with multi-tenant slicing in an ETSI-OSM orchestrated and ONOS-controlled IP over WDM transport network. OCIS codes: (060.4250) Networks; (060.4256) Network Optimization.
\end{abstract}

\section{Introduction}

Network Function Virtualization (NFV) promotes a paradigm shift in carrier networks allowing the replacement of specialized network hardware (i.e. middle-boxes) with virtualized network functions (VNFs) flexibly-deployed on commodity hardware appliances [1]. NFV permits the instantiation of network services, commonly referred as Service Chains (SC), by interconnecting VNFs either located in the same datacenter, or in several data centers across the transport network [2]. Indeed, NFV combined with Software-Defined Networking (SDN) enhances transport ecosystems with numerous optimization opportunities based on open initiatives [3]. For instance, ETSI's Open Source MANO (OSM) is an operator-lead NFV Management and Orchestration framework for NFV placement and life cycle control in datacenters, potentially connected by a transport network [4]. OSM orchestrates VNFs in one or more datacenters relying on Virtual Infrastructure Managers (VIMs) for managing the IT infrastructure, and defines a WAN Infrastructure Manager (WIM), as a software element abstracting and giving access to the SDN-enabled transport network via open programmatic interfaces.

In this context, the Metro-Haul project encompass the application of SDN/NFV technologies proposing scalable, dynamic and efficient metro networks to efficiently interface 5G access and high-capacity core/backbone networks [5]. Metro-Haul seeks the dynamic interconnectivity of two different types of nodes, Access Metro Edge Node (AMEN) and Metro Core Edge Node (MCEN), both with computational capabilities to permit SDN/NFV functionalities to provision VNFs close to the end users. Metro-Haul defines a Control Management and Orchestration $(\mathrm{COM})$ architecture [6] to offer ETSI-OSM network services in multiple geographically-located AMEN/MCEN with OpenStack VIMs [7], interconnected with an ONOS controller [8]. Both OpenStack and OSM provide multi-tenant capabilities permitting logical (and operational) isolation among different network carriers while sharing the same underlying infrastructure, which is referred to as slicing. Within the Metro-Haul scenario, we recently demonstrated the open-source planning tool Net2Plan [9] assisting an OSM instance in the optimal allocation and instantiation of network services (SCs) in a simulated transport network [10]. Subsequently, our demonstration in [11] included an ONOS controller to emulate the packet-layer network and considered the end-to-end latency requirements to perform the SC allocation, VNF instantiation and flow allocation.

The demonstration presented in this paper is based on two major components. The first component, namely IT resource manager, is a Net2Plan extension devoted to the interaction with OpenStack for permitting three functionalities: (i) multitenant IT slicing, (ii) advanced multi-VIM IT resource visualization and (iii) intra-VIM virtual machine (VM) migration. The second component, identified as carrier represents a network operator requesting the deployment of a network service so that Net2Plan assists OSM for the SC computation and NFV instantiation, and ONOS for the flow allocation. Additionally, this demonstration expands [11] emulating an IP-over-WDM transport network with ONOS, expanding the original single layer view.

\section{Demonstration Overview}

This demonstration is based on two major components implemented as Net2Plan extensions devoted to the following roles: IT resource manager and (potentially more than one) carrier. On the one hand, the IT resource manager employs the (new) open-source Net2Plan extension for interfacing multiple OpenStack instances which enables multi-tenant slicing, IT resource visualization and VM migration. On the other hand, the carrier leverages on our previous work [11] to deploy a network service performing SC computation and NFV instantiation. The network service is deployed, based on the assistance of the IT resource manager, transparently as a slice in a multi-tenant infrastructure (functionality (i) multitenant IT slicing) with awareness of the internal resources of the datacenters (functionalities (ii) advanced IT resource visualization and (iii) intra-cluster VM migration). An overview of the demonstration is shown Fig. 1(a), which contains the following components: 

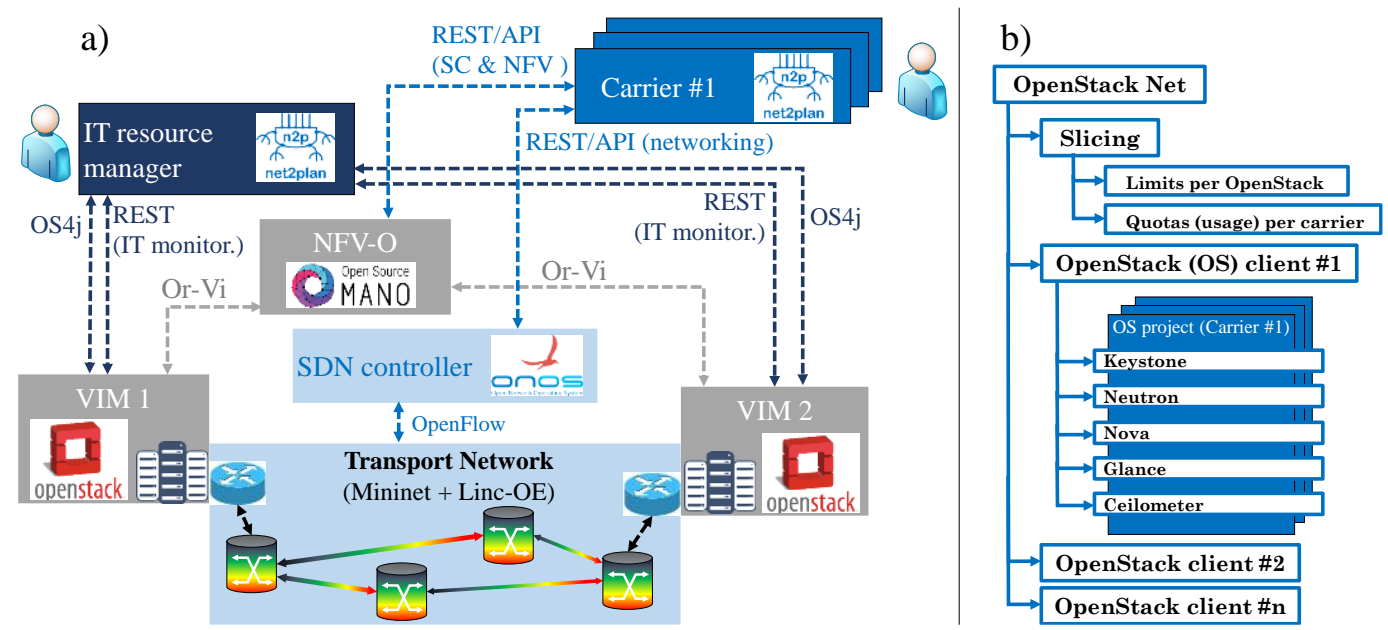

Fig. 1. (a) Demonstration architecture. (b) IT resource manager internal structure.

1. The IT resource manager is implemented as a Net2Plan plugin available in [12]. Its primary goal is permitting rapid algorithm development and prototyping considering the inner resources in OpenStack-managed data centers. The IT resource manager contains the following elements (see Fig. 1(b)). An OpenStackNet object groups and controls all the information gathered from a set of OpenStack clients, each of them interacting with an OpenStack instance (see interfaces description below). The OpenStackNet global view of the IT infrastructure permits the definition of the Slicing functionality based on the total available resources (Limits per OpenStack) and quotas definition per carrier (while also monitoring the quotas' usage in \%). OpenStackNet stores the quotas available for each project instantiated in each OpenStack client. A project in OpenStack is defined as a group of users which access to an isolated portion of the computing resources. We leverage on the OpenStack project concept to associate it with a carrier employing a logical partition of the computing resources.

Each OpenStack client interacts with its correspondent OpenStack instance employing two interfaces, illustrated as OS4j and REST (IT monitoring) in Fig. 1(a). OpenStack4j is a Software Development Kit (SDK) library to interact/interoperate with OpenStack via a Java language [13]. We use OpenStack4j to obtain the services' list available (Keystone, Nova, Neutron and Glance in our case) in each OpenStack instance and their internal (per service) functionalities. A proprietary REST API has been developed to employ advanced monitoring functionalities available in Gnocchi telemetry service, which expands OpenStack monitoring service's (Ceilometer) functionalities.

2. The Carrier $(s)$ module emulates the behavior of a network operator that requests the deployment of a network service through the GUI of Net2Plan. This module is a Net2Plan plugin based on our experience in previous demonstrations to perform SC computation which represents an evolution of the classical Path Computation Element (PCE) tuned for service chain allocations, where the path is constrained to traverse a sequence of VNFs [11]. The combination of path computation and VNF placement decision is implemented as an algorithm in this Net2Plan plugin. Additionally, the current implementation considers the IP-over-WDM emulation of the transport network.

3. The NFV-Orchestrator (NFV-O) is represented by OSM (release 3) and performs the management and orchestration of the virtualization infrastructure deploying VNFs leveraging on VIMs.

4. VIMs, implemented with OpenStack (Queens), are responsible for instantiating and hosting the VMs of the VNFs.

5. SDN-control of the metropolitan network is performed by ONOS (1.14) via OpenFlow both to the emulated IP switches in the Mininet network and the Linc-OE emulated WDM layer.

6. Emulated transport is provided by the combination of Mininet and Linc-OE so that realistic packet flows through a metropolitan-size IP network over an emulated WDM layer are established between the VNFs instantiated in commodity hardware appliances.

\section{Demonstration Testbed Configuration and Workflow}

This demonstration is composed of a personal laptop that runs two Net2Plan instances (IT resource manager and carrier modules) and OSM (in a VM); two mini-PCs that emulate AMEN/MCEN nodes in a metropolitan network with a VIM OpenStack instance in each of them; one mini-PC runs the ONOS SDN controller and the Mininet / LincOE emulated metropolitan IP-over-WDM transport network; and two regular auto-configured switches (one devoted to control-plane and the other devoted to data-plane). The demonstration showcases three features employing the IT resource manager module and a use-case using the carrier module. 


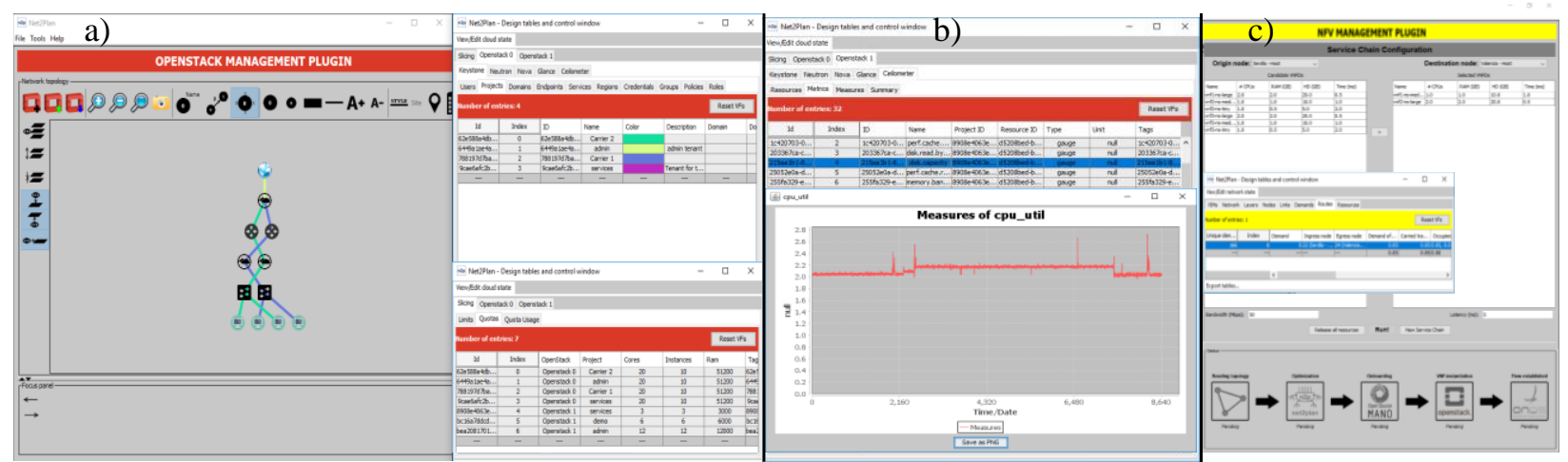

Fig. 2. Graphical User Interface (GUI) of the IT manager view (a) multitenant IT slicing and (b) IT resource visualization; and (c) carrier view.

- Multitenant IT slicing is demonstrated (see Fig. 2(a)) with the control of the IT infrastructure (VMs, networks and routers) by different carriers (represented with different colors, legend in the upper table) and according to a set of quotas (configured in the lower table).

- IT resource visualization, as shown in Fig. 2(b), exploits the developed REST API to obtain from the Gnocchi telemetry service the available list of resources (instances, networks, volumes, etc.) that generate metrics and measures. We process these information to provide a summary overview (including a graphical plot).

- Intra-cluster VM migration is permitted in our multi-node OpenStack cluster instantiation. Additionally, the IT resource manager plugin permits launching a terminal console from the GUI thus assessing the correct instantiation of the VMs.

- The carrier module executes the SC computation, NFV instantiation, and ONOS (IP-over-WDM) flow allocation in the sequence shown in Fig. 2(c). Specifically, (i) the topology is loaded in the GUI from ONOS and OSM, (ii) the optimization algorithm is executed within the Net2Plan plugin, (iii) the network service descriptor is sent to OSM, (iv) VMs are instantiated in the OpenStack instances, and (v) OpenFlow rules are established via ONOS.

\section{Innovation}

This demonstration combines two distinct roles implemented as Net2Plan plugins. A (new) IT resource manager module proves advanced joint control and management of multiple OpenStacks-based data centers in a metropolitan IP-over-WDM network. A carrier module, based on our previous experience [11], here extended with an IP-overWDM flavor, requests the deployment of an inter-node service chain that is internally optimized in the Net2Plan implementation for assisting OSM on the NFV instantiation, and ONOS for the flow allocation.

\section{OFC Relevance}

The proposed demonstration is in line with current trends in the OFC community, combining a carrier module relevant for topic N3 with an IT resource manager module relevant for the current revised topic N2, as a proof-of-concept in a dynamic SDN/NFV inter- and intra-data center environment optimized by the open-source Net2Plan tool.

\section{Acknowledgments}

This work was supported in part by the Spanish Government for the ONOFRE-2 project under Grant TEC2017-84423-C3-2-P and the Elastic Networks project under Grant TEC2015-71932-REDT, and by the European Commission for the H2020-ICT-2016-2 METRO-HAUL project (G. A. 761727) and the H2020-MSCA-IF-2016 INSPIRING-SNI project (G. A. 750611).

\section{References}

[1] M. Chiosi, et al., "Network functions virtualisation: An introduction, benefits, enablers, challenges and call for action," in Proc. SDN OpenFlow World Congr., Darmstadt, Germany, pp. 22-24, Oct. 2012. http://portal.etsi.org/NFV/NFV_White_Paper.pdf [acc. Nov 9 $9^{\text {th }} 2018$ ]

[2] J. G. Herrera, et al., "Resource allocation in NFV: A comprehensive survey," IEEE Trans. Netw. Service Manag., v.13(3), pp.518-532, 2016.

[3] M. Garrich, et al., "Open-source Network Optimization Software in the Open SDN/NFV Transport Ecosystem," JLT, 2018.

[4] Open source mano. https://osm.etsi.org/ [acc. Nov $9^{\text {th }} 2018$ ]

[5] Metro-Haul. https://metro-haul.eu/ [acc. Nov $\left.9^{\text {th }} 2018\right]$

[6] R. Casellas, et al., "Metro-haul: SDN control and orchestration of disaggregated optical networks with model-driven development," ICTON, 2018.

[7] OpenStack. https://www.openstack.org/ [acc. Nov $\left.9^{\text {th }} 2018\right]$.

[8] Open Network Operating System (ONOS) https://onosproject.org/ [acc. Nov $\left.9^{\text {th }} 2018\right]$

[9] Net2Plan. http://www.net2plan.com/ [acc. Nov $\left.9^{\text {th }} 2018\right]$

[10]F.J. Moreno-Muro, et al, "Joint Optimal SC Allocation, VNF instantiation and Network Resource Management Demonstration", $O F C, 2018$.

[11]F.J. Moreno-Muro, et al, "Latency-aware Optimization of SC Allocation with joint VNF instantiation and SDN network control", ECOC, 2018.

[12]Net2Plan - OpenStack plugin. https://github.com/girtel/Net2Plan-OpenStack [acc. Nov $9^{\text {th }} 2018$ ]

[13] OpenStack 4 Java. http://www.openstack4j.com/ [acc. Nov $9^{\text {th }}$ 2018] 